\title{
Efficient Resources Allocation for Femtocells in Heterogeneous Cellular Networks
}

\author{
Ruixue Liu ${ }^{1, a}$, Yang Chen ${ }^{2, b}$ and Xiaoling Xiong ${ }^{1, c}$ \\ ${ }^{1}$ Chongqing University of Posts and Telecommunications, Chongqing 400065, China \\ ${ }^{2}$ Chongqing Integrated Automation System Co Ltd, Chongqing 400000, China \\ a'839830867@qq.com, ${ }^{b} 412993472 @ q q . c o m,{ }^{c} 906084127 @ q q . c o m$
}

Keywords: Macrocell, femtocell, interference, throughput.

\begin{abstract}
We study a novel resources allocation approach for femtocells according to the quantity of femtocells and available channels in cognitive heterogeneous network. Due to the co-channel manner in macrocells and femtocells, femtocell base station (FBS) is combined with cognitive radio to obtain available spectrum resources and protect macrocell users (MUEs) from interference of femtocells. Then, the proposed channel allocation approach improves the throughput of femtocells and mitigates the co-tier interference among femtocells. Numerical results verify the conclusions.
\end{abstract}

\section{Introduction}

Although the macrocell has a large coverage area, it provides poor communication quality for indoor users. Therefore, a promising approach, which embeds femtocells in the present cellular system, has emerged to improve the performance gain of the system.

But it is critical to seek for available spectrum resources and make the best of scarce resources for CFs in dense networks. In [1], the CFs obtain the unoccupied sub-channel by estimating the energy of sub-channels in a group-wise manner and then allocate them with the lowest interference signature to FUEs. In [2], the sub-channel allocation scheme for orthogonal frequency division multiplexing access (OFDMA) system in Long Term Evolution (LTE) downlink is applied by considering the contiguous resource blocks (RBs) when the available resources are more than FUEs. The contiguous RBs allocation in [3] is performed to increase the femtocell throughput, however, RBs are still remained at the end of the assignment process.

From the above statement, RBs are still remained on the condition that the quantity of available RBs is more than the number of FUEs. So a small portion of spectrum resources is wasted. Therefore, we propose a dynamic channel allocation approach which is employed according to the number of available channels and FUEs accessing the system at the beginning of different time slot. And there are no spectrum resources remained.

\section{System Model}

We consider a dynamic downlink of an OFDMA system comprising $M$ hexagonally-shaped macrocells and $N$ femtocells at a time slot. The cross-tier interference from femtocell3 to MUE1 and the co-tier interference from femtocell2 to FUE1 are illustrated in Fig. 1. And a femtocell can serve only a FUE, which is randomly deployed in the coverage area with the radius of $R_{f}$.

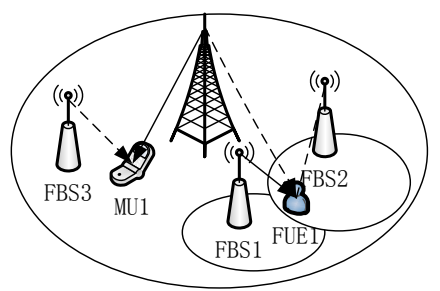

Figure 1: Interference scenarios in heterogeneous networks 
The allocated transmitting power of the FBS $k$, the adjacent FBS $j$ and the nearby macrocell base station (MBS) $i$ on the subcarrier $n$ are $P_{k}^{n}, P_{j}^{n}$ and $P_{i}^{n}$, respectively. The channel power gains from the adjacent FBS $j$, the nearby MBS $i$ and the FBS $k$ to FUE $k$ are $G_{j, k}^{n}, G_{i, k}^{n}$ and $G_{k}^{n}$, respectively. $\sigma_{n, k}^{2}$ denotes the noise power of additive white Gaussian noise (AWGN). The achievable instantaneous SINR of FUE served by FBS $k$ on the $n t h$ subcarrier can be expressed as

$$
\operatorname{SINR}_{k}^{n}=\frac{P_{k}^{n} G_{k}^{n}}{I_{c o, k}^{n}+I_{c r o s s, k}^{n}+\sigma_{n, k}^{2}},
$$

where $I_{c o, k}^{n}=\sum_{j=1, j \neq k}^{N} \alpha_{j}^{n} P_{j}^{n} G_{j, k}^{n}, I_{c r o s s, k}^{n}=\sum_{i=1}^{M} \alpha_{i}^{n} P_{i}^{n} G_{i, k}^{n}$ and $\sigma_{n, k}^{2}=N_{0} W$.

Herein, $\alpha_{j}^{n}$ and $\alpha_{i}^{n}$ respectively describe the utility function of femtocell $j$ and macrocell $i$ through the sub-channel $n$. If the sub-channel $n$ is allocated to femtocell $j$ and macrocell $i$, $\alpha_{j}^{n}=1$ and $\alpha_{i}^{n}=1$. Otherwise, $\alpha_{j}^{n}=0$ and $\alpha_{i}^{n}=0$.

Similarly, the achievable instantaneous SINR of MUE $u$ on the subcarrier $n$ can be given by

$$
\operatorname{SINR}_{u}^{n}=\frac{P_{u}^{n} G_{u}^{n}}{I_{c o, u}^{n}+I_{c r o s s, u}^{n}+\sigma_{n, u}^{2}},
$$

where $I_{c o, u}^{n}=\sum_{j=1, j \neq u}^{N} \alpha_{j}^{n} P_{j}^{n} G_{j, u}^{n}, I_{c r o s s, u}^{n}=\sum_{i=1}^{M} \alpha_{i}^{n} P_{i}^{n} G_{i, u}^{n}$ and $\sigma_{n, k}^{2}=N_{0} W$.

Moreover, for purpose of representing the overall performance preferably, we take the available transmission rate of the FUE $k$ on the $n t h$ sub-channel as an example, it can be given by [4]

$$
C_{k}^{n}=\frac{W}{N_{s c}} \sum_{k=1}^{N} \sum_{n=1}^{N_{s c}} \log 2\left(1+\operatorname{SINR}_{k}^{n}\right)
$$

Where, $N_{s c}$ is the number of sub-channels in the system?

\section{Cognitive Interference Management Scheme}

Due to the number variation of FUEs, the available channels are remaining or used up is unknown for the new FUEs at the next time slot. Therefore, the energy sensing scheme is introduced to obtain the available channel resources for femtocells by comparing the received signal power of a channel with an energy threshold and then deciding that the channel is available or occupied. Moreover, a detailed channel allocation procedure when the available channels are adequate for FUEs is applied to improve the capacity gain of the whole system.

CR-based energy detection: We assume that there are $x$ FUEs accessing the network at the beginning of the next time slot and $x$ is less than the total number $N_{s c}$ of sub-channels.

According to the theory of CR energy detection, the signal observed for the FBS is represented as:

$$
r(m)=h(m) s(m)+w(m)
$$

Herein, $r(m)$ is the signal received by the FBS, $s(m)$ denotes the signal transmitted by the MUE, $h(m)$ indicates the channel's gain from the MUE to the FBS, $w(m)$ is the AWGN sample. $m$ and $M$ respectively represent the sample index and the total number of samples. The average received signal energy is obtained by [4]

$$
R(M)=\frac{1}{M} \sum_{m=0}^{M-1}|r(m)|^{2}\left\{\begin{array}{l}
\geq \lambda, \text { occupied channels } \\
<\lambda, \text { idle channels }
\end{array}\right.
$$

A sub-channel is supposed to be occupied by the nearby MUEs if the received signal energy $R$ is more than a threshold $\lambda$. Otherwise, the sub-channel is idle. And the idle sub-channels are gathered in the set $A, N_{A}$ represents the number of available sub-channels. 
Sub-channel allocation for FUEs: We introduce the sub-channel allocation approach when the number of available sub-channels is more than the number of FUEs. The detailed allocation procedure is represented as follows:

Initialize: $A$ and $A$ ' respectively represent the sets of available channels and allocated channels, $F$ and $F^{\prime}$ respectively represent the sets of unallocated FUEs and completely allocated FUEs. At the beginning, $A=\left\{n_{1}, n_{2}, \ldots n_{N_{A}}\right\} \quad, \quad F=\left\{F_{1}, F_{2}, \ldots F_{x}\right\} \quad, \quad A^{\prime}=\varnothing \quad$ and $\quad F^{\prime}=\varnothing$. $\alpha_{i}^{j}=0, i=1,2, \ldots x, j=1,2, \ldots N_{A}$.

Step 1: Calculate $\operatorname{SINR}_{k}^{n}, k=1,2, \ldots X, n=1,2, \ldots N_{A}$ according to formula (1).

Step 2: Find the maximal $\operatorname{SINR}_{k}^{n}$ for FUE $k$ over the available sub-channel $n$.

Step 3: Allocate the sub-channel $n$ to the FUE $k$ and $\alpha_{k}^{n}=1$.

Step 4: Find the maximal $\operatorname{SINR}_{k}$ value between the adjacent sub-channel $n+1$ and $n-1$ for FUE $k$.

Step 5: For the sub-channel $n+1$ or $n-1$ with the higher SINR value, we calculate the current sum throughput $C_{k}, k=1,2, \ldots x$ when the channel $n+1$ or $n-1$ is allocated to each FUE temporarily. Then we allocate channel $n+1$ or $n-1$ to FUE $k$ with the maximal $\Delta C_{k}, \Delta C_{k}=C_{k}-C_{k}^{\prime}$. And $C_{k}^{\prime}$ represents the data transmission rate of FUE $k$ without the sub-channel $n+1$ or $n-1$. If no further capacity benefit for FUE $k$, update the $A, A^{\prime}, F, F^{\prime}$ and $\alpha_{k}^{n}$. Then carry out step 2. Otherwise repeat the step 4 and 5.

Step 6: If $A=\varnothing$ and $F=\varnothing$, the remaining sub-channels are allocated to their left-hand or right-hand FUEs, depending on which FUEs have a higher $\Delta C$ value.

\section{Simulation Results}

In this section, we present simulation results obtained by using proposed schemes to illustrate system performance, as shown in Figure 2 to Figure 3. System simulation parameters are presented in Table $\mathrm{I}[5]$. In the simulation, the $\lambda$ is set as $10 \mathrm{~dB}$.

\section{TABLE I SIMULATION PARAMETERS.}

\begin{tabular}{|c|c|}
\hline Parameter & Value \\
\hline The coverage radius of macrocell $R m$ & $1000 \mathrm{~m}$ \\
\hline The coverage radius of femtocell $R f$ & $20 \mathrm{~m}$ \\
\hline$N s c$ & 40 \\
\hline Path loss MBS-macrouser $R(\mathrm{~m})$ & $L=15.3+37.6 \log 10(R)$ \\
\hline Path loss FBS-femtouser serving link $R(\mathrm{~m})$ & $L=38.46+20 \log 10(R)+0.7(R)$ \\
\hline Path loss FBS-femtouser interference link $R(\mathrm{~m})$ & $L=15.3+37.6 \log 10(R)+\mathrm{pLhw}, \mathrm{pLhw}=20$ \\
\hline Path loss otherwise $R(\mathrm{~m})$ & $L=15.3+37.6 \log 10(R)+\mathrm{pLhw}, \mathrm{pLhw}=10$ \\
\hline Noise power spectral density $N 0$ & $-174 \mathrm{dBm} / \mathrm{Hz}$ \\
\hline Total channel bandwidth W & $10 \mathrm{MHz}$ \\
\hline
\end{tabular}

The cumulative distribution function (CDF) of the FUEs' signal to interference plus noise power ratio (SINR) is illustrated in Fig. 2, when the number of femtocells accessing the network at the beginning of next time slot is 20. Obviously, the proposed scheme achieves higher FUEs' SINR in contrast to the random sub-channel allocation scheme. This implies that the proposed scheme mitigates the co-tier interference efficiently.

Obviously, in Fig. 2, the proposed scheme improves the SINR performance of FUEs. Moreover, the CR-based energy detection scheme is able to find out more available spectrum resources on the whole bandwidth. And the proposed sub-channel allocation scheme seeks for the sub-channel with the best gain for each FUE. Therefore, the spectrum efficiency of FUEs is improved as seen in Fig. 3. 


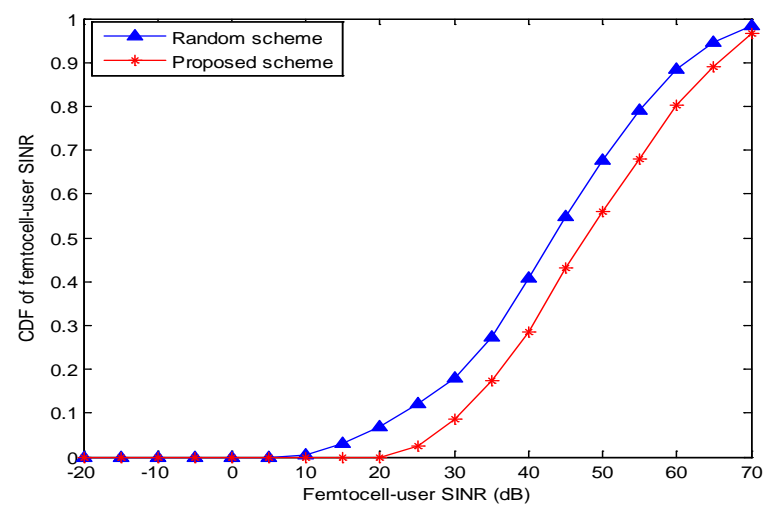

Fig. 2 The CDF of FUEs' SINR.

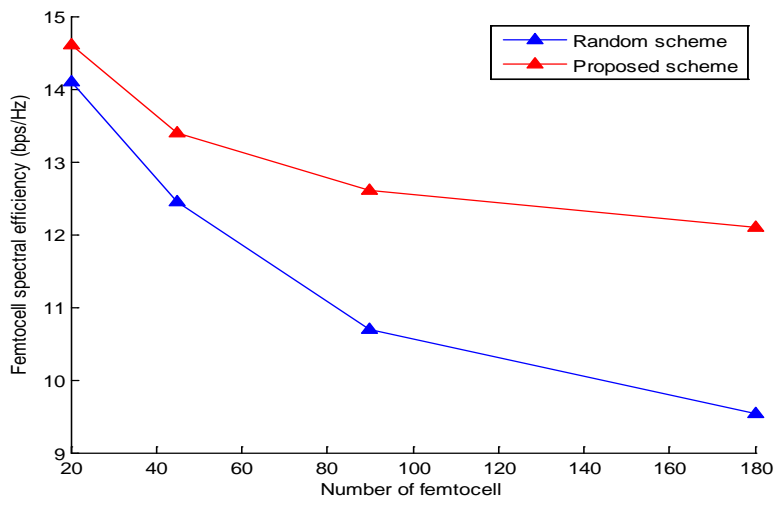

Fig. 3 The femtocell spectral efficiency.

\section{Summary}

In this paper, we focus on the condition that the available sub-channels are adequate the new FUEs at a time slot. The optimal sub-channel allocation approach achieves system capacity performance enhancement. An accurate sub-channel sensing scheme guarantees that femtocells are capable to find out available spectrum resources. As a consequence, the proposed scheme efficiently enhance the throughput and mitigate the cross-tier and co-tier interference of the whole system.

\section{References}

[1] Dong-Chan Oh, Heui-Chang Lee, and Yong-Hwan Lee. Cognitive Radio Based Femtocell Resource Allocation. International Conference on Information and Communication Technology Convergence, pp. 274 - 279, Nov. 17-19, 2010.

[2] Miao Wang, Zhangdui Zhong, and Qiuyan Liu. Resource Allocation for SC-FDMA in LTE Uplink. Proceedings of IEEE International Conference on Service Operations, Logistics and Informatics, pp. 601 - 604, 2011.

[3] Ching-Hsu Chang, Hsi-Lu Chao, and Chia-Lung Liu. Sum Throughput-Improved Resource Allocation for LTE Uplink Transmission. IEEE 74th Vehicular Technology Conference, 2011.

[4] Gang Ning, Qinghai Yang, Kyung Sup Kwak, and Hanzo, L. Macro- and Femtocell Interference Mitigation in OFDMA Wireless Systems. IEEE Global Communications Conference, pp. 5068-5073, Dec 3-7, 2012.

[5]Zhang Jian, and Zhu Qi. A Novel Adaptive Resource Allocation Algorithm for Multiuser OFDM-Based Cognitive Ra dio Systems. Proceedings of International Conference on Network Computing and Information Security,Vol. 2, pp. 442 -445, 2011.

[6] Yang-Yang Li, Sousa, and E.S.Cognitive Uplink Interference Management in 4G Cellular Femtocells. IEEE 21st International Symposium on Personal Indoor and Mobile Radio Communications, pp. 1567 - 1571, 2010. 